\title{
Diseño de un t-MOOC para la formación en competencias digitales docentes: estudio en desarrollo (Proyecto DIPROMOOC)
}

\author{
Design of a t-MOOC for training in teaching digital competences: \\ study under development (DIPROMOOC Project)
}

RECIBIDO 9/1/2019 ACEPTADO 19/1/2020 PUBLICAD0 1/6/2020

\author{
Julio Cabera-Almenara \\ Departamento Didáctica y Organización Educativa, Universidad de Sevilla, España \\ cabero@us.es \\ Rosalía Romero-Tena \\ Departamento Didáctica y Organización Educativa, Universidad de Sevilla, España \\ rromero@us.es
}

\section{RESUMEN}

Los MOOC se han convertido en una tecnología emergente que se está acercando fuertemente a las instituciones educativas. Su evolución permite identificar una diversidad de modelos. Los denominados t-MOOC son objeto de este artículo por su tendencia a apoyarse en la realización de tareas por parte del estudiante. La presencia de tecnologías en las tareas educativas hace que las competencias que deban poseer los docentes sean más amplias que el mero dominio de contenidos y metodologías de enseñanza, por lo que se hace necesario hacer hincapié en el desarrollo de la competencia digital docente (CDD). El proyecto que se presenta pretende analizar las posibilidades educativas de los t-MOOC para la formación de la CDD del profesorado. La investigación se articula en tres grandes fases: A) la primera es la concreción de los contenidos de una acción formativa centrada en la adquisición de la CDD. La unidad de contenido estará estructurada por una guía de aprendizaje: objetivos y competencias con recomendaciones para su seguimiento, bibliografía básica, bibliografía complementaria, un clip de vídeo y e-actividades que deberán desarrollar los estudiantes. B) La segunda fase es la creación de una comunidad virtual formada por profesorado de infantil/primaria, secundaria/bachillerato/formación profesional y universidad e investigadores preocupados por las CDD. C) Y la tercera la creación de un entorno formativo bajo la arquitectura t-MOOC, que constará de varias etapas: diseño y elaboración de los contenidos, producción de los contenidos del t-MOOC y evaluación de los contenidos y t-MOOC elaborado. Para esta última etapa se llevará a cabo una evaluación por los productores, por expertos, y se realizará un estudio piloto. Utilizaremos varios instrumentos tanto de corte cuantitativo (TAM, IMMS y TCC) como cualitativo (entrevistas a los participantes de las acciones formativa, y el análisis de contenido redes sociales creadas en el t-MOOC).

PALABRAS CLAVE competencia digital docente, t-mooc, formación profesorado, diseño de medios, evaluación de medios.

\section{ABSTRACT}

MOOCs have become an emerging technology that is very close to educational institutions. Its evolution allows to identify a diversity of models. The so-called t-MOOCs are the object of this paper because of their tendency to rely on the performance of tasks by the student. The presence of technologies in educational tasks means that the competences that teachers 
must possess are broader than the mastery of content and teaching methodologies, so it is necessary to emphasize the development of teacher digital competence (TDC). This project aims to analyze the educational possibilities of t-MOOCs for the training of teachers' TDC. The research is divided into three main phases: A) the first is the concretion of the contents of a training activity focused on the acquisition of digital teaching competence. The content unit will be structured by a learning guide: objectives and competencies with recommendations for follow-up, basic bibliography, complementary bibliography, a video clip and e-activities that require student development. B) The second phase is the creation of a virtual community formed by teachers of Childhood/Primary, Secondary/Baccalaureate/Professional Training and University Professors and Researchers concerned with TDC. C) The third and las part consist in creating of a training environment under the $\mathrm{t}-\mathrm{MOOC}$ architecture that will consist of several stages: design and elaboration of the contents, production of the contents of the t-MOOC and evaluation of the contents and t-MOOC developed. For this last stage, the producers and experts will carry out an evaluation and pilot study. It will be use several instruments both quantitative (TAM, IMMS and CBT) and qualitative (interviews to participants in training activities, and social media content analysis created in the t-MOOC).

KEYWORDS teacher digital competence, t-mooc, professional training, media design, media evaluation.

\section{INTRODUCCIÓN}

\subsection{La tecnología de los MOOC}

Desde que se realizó el primer curso considerado como MOOC en el 2008, denominado "Connectivism and Connected Knowledge (CCK08)", impartido por Downes y Siemens de la Universidad de Manitoba, estos se han convertido en una tecnología emergente que, en los últimos años, se está fuertemente consolidado. Y pasado ya el tiempo romántico de considerarlo como una tecnología disruptiva que transformaría la formación y las instituciones que a ellas se dedican, la realidad es que en la actualidad se están buscando vías que los concreten como una herramienta a utilizar en diversas acciones educativas. Ello está repercutiendo para que aumenten progresivamente estudios, publicaciones e investigaciones al respecto. Hecho que hace que sean considerados como una tecnología complementaria al sistema educativo formal y con verdaderas posibilidades para favorecer una formación flexible que facilite a la persona la compatibilidad de la formación con otro tipo de situaciones (Fundación Telefónica, 2015). MOOC puede definirse como cursos gratuitos

en abierto, compuesto fundamentalmente por Recursos Educativos Abiertos (OER) y diseñado para poder ser cursado, a través de una plataforma o entorno personal de aprendizaje instalado en la red Internet, por cualquier persona, de manera autónoma, sin necesidad de contar con un profesor o tutor de apoyo en red al otro lado de la conexión. (Marauri, 2014, p. 40)

Cursos que, de acuerdo con Castaño y Cabero (2013), presentan las siguientes características: es un recurso educativo que tiene cierta semejanza con una clase, con un aula; con fechas de comienzo y finalización; cuenta con mecanismos de evaluación; es online; de uso gratuito; es abierto a través de la web; no tiene criterios de admisión y permite la participación interactiva a gran escala de cientos de estudiantes.

Su evolución ha permitido identificar una diversidad de modelos: transferMOOCs, madeMOOCs, synchMOOCs, etc. como recientemente han apuntado Escudero-Nahón y Núñez-Urbina (2020). Realmente los podemos concretar en dos tipos básicos: xMOOC y cMOOC (Cabero et al., 2014a); los primeros, tienden a ser cursos universitarios tradicionales de e-learning que se adaptan a las características de las plataformas de los MOOC. Mientras que los segundos se apoyan en la filosofía del aprendizaje conectivista de Siemens y 
Downes. Recientemente diferentes autores (Cabero et al., 2014b; Martí, 2012; Scopeo, 2013; Vázquez et al., 2013) amplían esta división con un modelo que se podría considerar como híbrido de los dos anteriores y que tiende a apoyarse en la realización de tareas por parte del estudiante: los denominados t-MOOC, -MOOC o task-based MOOC. Este tipo de MOOC presenta, frente a las otras modalidades, una serie de ventajas: implican acciones más significativas para los estudiantes, no son meros transmisores de información, no tratan al alumno de forma masiva, o centrase exclusivamente en las conexiones que realizan los alumnos puede conllevar la no producción de aprendizaje significativo. Para Osuna-Acedo et al. (2018) estos MOOC se apoyan en una serie de aspectos como son: áreas auténticas, transferencia del aprendizaje hacia la profesión, transformación pedagógica, TRIC (tecnologías + relación + información + comunicación), transmedialidad, temporalidad abierta, transnacionalismo, talento interpretativo, trabajo colaborativo y tolerancia.

Por otro lado, debemos señalar que los MOOC están tendiendo a cursos más personalizados o POOC (personalized open online courses), a SPOOCs (self-paced open online course) y a NOOCs (nano cursos abiertos, masivos y en línea); estas últimas modalidades tienden a potenciar más la participación de los estudiantes en la construcción colectiva del conocimiento, al mismo tiempo reclaman un compromiso alto y demandan un esfuerzo alto de seguimiento y tutoría, especialmente para resolver debates.

Las ventajas que nos ofrecen estas nuevas visiones de estos cursos es que permiten una formación más participativa y personalizada, evitando caer en lo que se llama la "MacDonalización” de la educación, y permiten mantener un contacto continuo con participantes retrasados o que no avanzan al ritmo esperado.

En contraposición, los errores más comunes que se apuntan respecto a su calidad educativas vienen de diferentes aspectos como son: baja calidad de los diseños instrucciones (Calvo et al., 2016; Margaryan et al., 2015; Sosa y Fernández, 2015) estar muy apoyados en una metodología tradicional transmisiva de información y apoyada en vídeos busto parlantes (Fernández-Díaz et al., 2017; Luján, 2015) y en aportar pocos recursos (Duart, 2017). A ellos debemos incorporar la problemática de la evaluación (Dawna et al., 2014; Sánchez et al., 2017), respecto a la cual se utilizan por lo general dos tipos de procedimientos: evaluación automática y por pares; si bien, como se está poniendo de manifiesto últimamente, cada vez están surgiendo nuevos planteamientos (Sánchez y Prendes, 2015; Sánchez et al., 2017).

Por lo que se refiere a sus limitaciones, algunas de ellas han sido apuntadas al reconocer que se han desarrollado más análisis técnicos y teóricos, reflexiones sobre el marketing y deliberaciones filosóficas; que investigaciones educativas sobre la calidad de los mismos (Cabero et al., 2016; Duart, 2017, León-Urritia et al., 2018; Mengual y Roig, 2015) que no han aportado resultados muy significativos.

Sin embargo, si hay algunos de aspectos que se han presentado como significativos para que los alumnos muestren un elevado grado de aceptación de esta tecnología: la satisfacción por la reputación del curso y la apertura percibida, la capacidad que el alumno tenga de autorregular su aprendizaje (García Barrera et al., 2017), el aumento del nivel de participación del estudiante mediante el uso de redes sociales y herramientas de comunicación como los foros (Castaño et al., 2017; González y Carabantes, 2017; Ruiz-Palmero et al., 2019), la utilización de vídeos introductorios e incorporados a la estructura de la lección (González y Carabantes, 2017; Hansch et al., 2015; Sánchez et al., 2017), y hacer un fuerte uso de las tareas (Castaño et al., 2014).

Otro aspecto importante a considerar, relacionado con variables psicológicas que se han mostrado significativas para explicar el éxito en estas acciones, son la motivación que posea el estudiante, su grado de aceptación de esta tecnología, su percepción de control conductual, la percepción de éxito, la metacognición o las actitudes hacia los MOOC (Jung y Lee, 2018; Quiliano-Terreros et al., 2019; Watted y Barak, 2018; Zhou, 2016). 
Finalmente, indicar que se han construido diferentes instrumentos para su evaluación que indirectamente ofrecen pistas para su diseño y utilización (CRUE, 2015; Guerrero, 2015; Mengual y Roig, 2015), a su planificación/gestión, el diseño de aprendizaje, la comunicación-interacción, la organización y estructura de los contenidos, los recursos didácticos, la capacidad de motivación, la estructura multimedia o el estilo del lenguaje. Debe estar bien documentada (más del 60\% de las citas deben hacer referencia a literatura actual) y contener planteamiento del problema o tema objeto de estudio, antecedentes y fundamentación teórica. Los objetivos de la investigación deben ser establecidos de forma clara.

\subsection{Las competencias digitales docentes}

Pocas son las palabras que se utilizan en mayor grado que el término competencia, y todavía más si lo relacionamos con otros como profesionales, docentes o digitales. López (2016), tras realizar una revisión de diferentes usos, llega a una síntesis indicando que la competencia

vendría a ser una actuación integral capaz de articular, activar, integrar, sintetizar, movilizar y combinar los saberes (conocer, hacer y ser) con sus diferentes atributos." Referidas a los docentes se refieren al conjunto de conocimientos, características personales, actitudes y habilidades que posibilitan el desempeño de la actuación docente; "es decir hablamos de competencia si hay desempeño, conocimiento y acciones. (Tourón et al., 2018: 27)

En la actualidad la fuerte presencia de las tecnologías de la información y la comunicación, en la sociedad en general y en las instituciones educativas en particular, hace que las competencias que deban poseer los docentes sean más amplias que el dominio de los contenidos y las metodologías de enseñanza, y tenga que referirse necesariamente a las tecnologías que se incorporan en la acción de la enseñanza; es decir a la CDD.

Competencia que ha sido reclamada, tanto por diferentes instituciones como la Comisión Europea (2013), que la considera como una competencia para el uso crítico y seguro de las TIC, el Informe DIGCOMP (Ferrari, 2013) que la identifica como una de las competencias transversales que debe poseer toda persona para desenvolverse en la sociedad del conocimiento, o el Instituto Nacional de Tecnología Educativa y de Formación del Profesorado (INTEF, 2017). Y desde el ámbito de la teoría y la investigación educativa, donde se llama la atención para que el docente se encuentre capacitado en las mismas (Castañeda et al., 2018; Durán et al., 2016a, 2016b y 2018; Gisbert y Lázaro, 2015; Hatlevik, 2016). La base de su significación nos la encontramos en una serie de hechos, como son: la significación que las TIC están adquiriendo en la sociedad y en las instituciones educativas, la exclusión social producida por el hecho de no tener acceso a la información; sin olvidarnos que su no adquisición repercute directamente en el bajo uso educativo de las TIC y en usos muy tradicionales y no innovadores (Sosa et al., 2018; The Scottish Government, 2016; Unesco, 2016).

Por lo que se refiere a las dimensiones sobre las que se puede centrar la adquisición de la CDD se han indicado diferentes propuestas; así, para el INTEF (2017) estas deben centrarse en las cinco siguientes: a) información y alfabetización informacional, b) comunicación y colaboración, c) creación de contenido digital, d) seguridad y e) resolución de problemas; por su parte Rangel (2015), tras revisar distintas instituciones como la Unesco (2008), ISTE (2008), etc., propone que la CDD debe centrarse en: 1) manejar conceptos y funciones básicas de la computadora, 2) realizar tareas básicas de conectividad, instalación y seguridad del equipo de cómputo, 3) manejar funciones básicas de los programas de productividad, 4) mostrar una actitud positiva para su actualización permanente en temas relacionados con las TIC, 5) saber cómo localizar y recuperar 
información, 6) analizar y seleccionar la información de manera eficiente, 7) organizar la información recuperada de Internet de manera adecuada y 8) utilizar y presentar la información de manera eficaz, ética y legal. Por su parte, para el contexto latinoamericano, Lázaro et al. (2018) nos hablan de una serie de dimensiones con una diversidad de subdimensiones cada una de ellas, en concreto: Didáctica, curricular y metodológica (planificación docente y competencia digital, las tecnologías digitales como facilitadoras del aprendizaje, tratamiento de la información y creación de conocimiento, atención a la diversidad: necesidades educativas especiales (NEE), evaluación, tutoría y seguimiento de los estudiantes, y línea metodológica de la unidad académica). Planificación, organización y gestión de espacios recursos tecnológicos digitales (ambientes de aprendizaje, gestión de tecnologías digitales y aplicaciones, espacios con tecnologías digitales de la unidad académica, proyectos de incorporación de las tecnologías digitales, e infraestructuras tecnológicas digitales). Relacional, ética y seguridad (ética y seguridad, inclusión digital, comunicación, difusión y transferencia del conocimiento, contenidos digitales y comunidad educativa, e identidad digital de la institución). Personal y profesional (acceso libre a la información, creación y difusión de material didáctico con licencias abiertas, liderazgo en el uso de las tecnologías digitales, formación permanente, comunidades de aprendizaje virtuales: formales, no formales e informales, entorno personal de aprendizaje, e identidad y presencia digital).

O la propuesta del DigCompEdu (Cabero y Palacios, 2020), del marco europeo, que es en la actualidad una de las más utilizadas.

\section{MATERIAL Y MÉTODO}

El Proyecto DIPROMOOC pretende analizar las posibilidades educativas que, para contextos de formación del profesorado en competencias digitales pueden tener los MOOC y, más concretamente, los t-MOOC. El análisis se efectuará desde diferentes perspectivas, tanto tecnológicas-instrumentales como educativas, de diseño de entornos formativos, hasta la referida a la formación y el perfeccionamiento del profesorado.

\subsection{Objetivos}

Los objetivos que se plantean en el proyecto DIPROMOC son:

1. Analizar las posibilidades, potencialidades y dificultades que ofrecen lost-MOOC para su incorporación como tecnologías formativas para los docentes.

2. Creary evaluar un entorno formativo bajo la arquitectura t-MOOC, para la capacitación del profesorado de infantil/primaria, secundaria/bachillerato/formación profesional y universitario en la adquisición de la CDD.

3. Conocer el grado de motivación, nivel de satisfacción, carga cognitiva empleada en el procesamiento de la información y grado de aceptación de la tecnología que despierta en los docentes la participación en una acción formativa en formato t-MOOC.

4. Analizar las posibles influencias que el grado de motivación, nivel de satisfacción, grado de aceptación de la tecnología y carga cognitiva invertida por el docente en la acción formativa, tienen en la adquisición de los conocimientos presentados en el t-MOOC, referidos a la CDD.

5. Determinar los contenidos formativos que debe contener una acción formativa centrada en la 
adquisición de la competencia digital del profesorado de infantil/primaria, secundaria/bachillerato/ formación profesional y universitario en la adquisición de la CDD.

6. Analizar las posibilidades que los t-MOOC presentan para la adquisición de los conocimientos referidos a la CDD expuestos en los t-MOOC diseñados.

7. Poner en acción y validar el entorno producido bajo la arquitectura t-MOOC para la capacitación del profesorado en la adquisición de la competencia digital.

8. Indagar sobre las dificultades técnicas, curriculares y organizativas que pudieran presentarse a la hora de organizar una acción formativa apoyada en la tecnología t-MOOC.

9. Crear una comunidad virtual formada por profesorado e investigadores preocupados por las competencias digitales que deben poseer los profesores en los diferentes niveles educativos.

10. Analizar la significación que las redes sociales y las herramientas de interacción presentadas en la acción formativa soportadas en el t-MOOC, tienen para la significación de la acción formativa.

11. Analizar la relación entre la motivación, nivel de satisfacción, carga cognitiva invertida y grado de aceptación de la tecnología, y el volumen de mensajes ofrecidos en las redes sociales y herramientas de interacción incorporadas en la acción formativa.

12. Analizar si las competencias digitales docentes a adquirir por el profesorado son similares para los docentes de infantil/primaria, secundaria/bachillerato/formación profesional y de universidad.

\subsection{Estructura de la investigación}

La investigación se articula en tres grandes fases:

Primera fase: determinación de los contenidos formativos de una acción formativa centrada en la adquisición de la CDD.

Para alcanzar esta primera fase se realizarán diferentes actividades:

1.1 Revisión de las principales propuestas realizadas sobre las competencias digitales que deben poseer los docentes universitarios.

1.2 Realización de una lista de chequeo (check list) que recoja las principales dimensiones de la CDD que deben poseer los docentes de diferentes niveles educativos y los distintos niveles de adquisición propuesto.

1.3 Elaboración de la versión en Internet del instrumento elaborado en la etapa anterior. Para ello crearemos un instrumento ad hoc que será aplicado vía Internet, para facilitar su administración y recogida de información.

1.4 Para la evaluación de la lista y selección definitiva de las competencias digitales docentes llevaremos a cabo un estudio Delphi mediante el juicio de expertos con profesionales de diferentes universidades españolas y latinoamericanas (Barroso y Cabero, 2010). La validez de la técnica del juicio de experto viene determinada, fundamentalmente, por las características que posean los expertos seleccionados. En nuestro caso, para asegurarnos su pertinencia, estableceremos inicialmente una serie de características para su selección: que sean profesores de tecnología educativa y TIC 
aplicadas a la educación, y personal técnico de los secretariados y servicios de recursos educativos de diferentes instituciones educativas (CEP) y universidades; al mismo tiempo, se aplicará el coeficiente de competencia experta (Cabero y Barroso, 2013) para su selección definitiva.

Segunda fase: crear una comunidad virtual formada por profesorado de infantil/primaria, secundaria/bachillerato/formación profesional, universidad e investigadores preocupados por la CDD.

Nuestra experiencia en investigaciones anteriores nos llevó a pasar por diferentes tipos de redes sociales: Grouply, Elgg, Ning, Google+; decantándonos finalmente por la última por diferentes motivos: facilidad de manejo, familiaridad de la gran mayoría de personas con ella, posibilidades que ofrece, comodidad, y estabilidad. La red será cerrada y solo se podrá participar en ella mediante invitación, el motivo de ello es evitar la penetración de instituciones comerciales. Su análisis se llevará a cabo mediante un programa que aún no se ha elegido.

Tenemos que señalar que la comunidad virtual creada nos servirá también como instrumento para dar a conocer los datos progresivos que se vayan obteniendo del proyecto y obtener también un feedback respecto a la evolución del proyecto, la validez de los resultados alcanzados y el interés despertado por el t-MOOC que se elaborará.

Tercera fase: crear un entorno formativo bajo la arquitectura t-MOOC para la capacitación del profesorado de infantil/primaria, secundaria/bachillerato/formación profesional y universidad en la adquisición de la competencia digital docente.

En esta tercera fase se persigue crear un curso de formación del profesorado en CDD bajo la arquitectura t-MOOC y, para ello, se realizarán diferentes acciones que se detallan a continuación.

3.1 Producción de los contenidos.

Una vez identificados los contenidos a desarrollar en el t-MOOC mediante la técnica del juicio de experto, y determinada la estructura del mismo, se pasará a su distribución entre los diferentes miembros del equipo de investigación. Para asegurar que todos los bloques de contenidos tengan la misma estructura se elaborará un libro de estilo donde se plantea la estructura general, los criterios de calidad a seguir, e-actividades a realizar, etc.; para ello nos apoyaremos en el libro de estilo de diseño de materiales para la formación virtual ya elaborado por nosotros (Cabero et al., 2014b) y en el "Informe MOOC y criterios de calidad" elaborado en el 2015 por la CRUE.

La estructura que dispondrán los t-MOOC será la siguiente: presentación de la acción formativa, que se llevará mediante un clip de vídeo elaborado en formato polimedia (Cabero, 2018) y con una guía del curso (se indicará el cronograma, el programa y los criterios de evaluación), el desarrollo, que comenzará con dos actividades básicas, un tiempo para que los estudiantes se socialicen (se presenten, muestren sus interés por el curso,...) en un foro especialmente creado para ello, su tiempo será de una semana; posteriormente, vendrá el período de trabajo con los contenidos, teniendo cada una de las unidades que corresponderán con las diferentes CDD identificadas la siguiente estructura: 1) guía de aprendizaje: objetivos y competencias que se desean alcanzar con esa unidad, recomendaciones para su seguimiento, bibliografía básica, bibliografía para saber más; 2) clip de vídeo presentando la información básica de la unidad mediante un polimedia; 3) e-actividades que deberán desarrollar los estudiantes (se propone la realización de dos actividades como máximo de las cuatro propuestas). La 
forma en la cual se evaluarán las actividades serán las utilizadas en los MOOC. Para la elaboración de los vídeos se tendrán en cuenta las recomendaciones ofrecidas por diferentes autores para la producción de estos recursos cuando son incorporados a los MOOC: corta duración, evitar los bustoparlantes, incorporar entusiasmo, etc. (Cabero, 2018; Guo et al., 2014; López, 2017; Meseguer, 2017).

3.2 Producción de los contenidos del t-MOOC.

Una vez elaborados los guiones de contenidos para cada uno de los proyectos, y revisados por los diferentes equipos de los proyectos coordinados con los técnicos, diseñadores gráficos y equipo de elaboración audiovisual, se realizarán los diferentes contenidos seleccionados. Para ello se utilizará el software de producción que permita que los contenidos puedan ser elaborados en formato SCORM (sharable content object reference model), que permite crear objetos pedagógicos estructurados y que puedan ser intercambiables, lo que facilitará su distribución a través de las diferentes plataformas MOOC (Miriadax, Edx,..) existentes, incluso en plataformas tradicionales de e-learning como Moodle.

Para la producción de los materiales se seguirán las etapas que tradicionalmente se siguen en la elaboración de cualquier tecnología: diseño, producción, postproducción y evaluación.

Señalar que, una vez construido los contenidos, se ubicarán en un servidor y se realizará una primera evaluación de los mismos por parte de los diferentes miembros de los equipos coordinados, en lo que se denomina como "autoevaluación por los productores".

3.3 Evaluaciones de los contenidos y t-MOOC elaborado.

Para la evaluación de los contenidos seguiremos diferentes procedimientos secuenciados:

a) Evaluación por los productores.

b) Evaluación por expertos

c) Estudio piloto.

Una vez adaptados los cambios identificados en las dos evaluaciones previas, se llevará a cabo un estudio piloto en diferentes universidades con el objeto de facilitar la generalización de los resultados. Los sujetos objeto del estudio piloto irán desde alumnos universitarios que se matricularán en un curso específico organizado por las diferentes universidades, hasta profesorado universitario en acciones formativas que se puedan llevar a cabo a través de los ICE de las respectivas universidades y profesorado de secundaria/ bachillerato/formación profesional, así como profesorado de infantil/primaria.

De forma específica se pretenden realizar tres acciones formativas que serán efectuadas en cada una de las sedes de los tres proyectos coordinados.

Para su evaluación utilizaremos tanto una metodología cuantitativa como cualitativa. La primera la llevaremos a cabo mediante una investigación de tipo cuasiexperimental (Hernández et al., 2010), donde recogeremos información de diferentes variables: adquisición de la información de los contenidos presentados en el t-MOOC, nivel de satisfacción y aceptación de la tecnología por los participantes en la acción formativa, motivación y la evaluación de la calidad de los materiales producidos. Y la segunda mediante entrevistas a los participantes en las acciones formativas y el análisis de contenido de las discusiones que se produzcan en las redes sociales expresamente creadas en el t-MOOC para la formación por pares. 
Con nuestro estudio queremos saber si los participantes en la acción formativa del t-MOOC, aprendían la información y conceptos que se presentaban en los mismos respecto a la CCD. Entendiendo en este caso por aprender la capacidad de recordar, comprender y aplicar la información presentada en los contenidos, y entendiendo por estas categorías lo especificado en la taxonomía de Bloom para la era digital (Chursches, 2008). El instrumento para aplicar será del tipo de elección múltiple y se administrará en la modalidad de pretets y postests.

Para el diagnóstico del nivel de aceptación y de satisfacción de la tecnología de los MOOC por el estudiante utilizaremos la adaptación realizada por Wojciechowski y Cellary (2013) del cuestionario "Modelo de Aceptación de Tecnología" (TAM) propuesto inicialmente por Davies et al. (1992) que ya sido aplicado a los MOOC (Ju et al., 2018), y que nosotros aplicamos a la realidad aumentada (Cabero et al., 2018).

En el modelo TAM la aceptación de la tecnología está representada por intención de utilizarla, que está determinada por la actitud del usuario hacia su uso y la utilidad que percibe de ella. Como señalan los autores del instrumento, la actitud hacia el uso de una tecnología está determinada por la percepción de la utilidad y facilidad de uso del sistema de los usuarios y por la facilidad de uso percibida. Además, la utilidad percibida y la facilidad de uso percibida, pueden estar afectadas por diversas variables externas, como las características del usuario y las de la tecnología y el entorno en el que se utiliza el sistema.

Diferentes metaanálisis realizados (López-Bonilla y López-Bonilla, 2011) han puesto claramente de manifiesto que el modelo TAM es un modelo válido y robusto para explicar la intención de uso de cualquier entorno tecnológico.

Al mismo tiempo hay que indicar que se ha mostrado como un modelo muy potente cuando ha sido analizado mediante el sistema de ecuaciones estructurales (Cabero y Pérez, 2018).

El diagnóstico de la motivación que había despertado la participación en la experiencia por los alumnos, lo efectuaremos mediante la "Encuesta de Motivación Materiales de Instrucción” (IMMS) construida por Keller (2010); el instrumento considera cuatro factores de motivación: atención, interés, confianza y satisfacción, y de forma concreta ha sido utilizado para conocer la motivación que despertaba la participación en un curso MOOC en los estudiantes (Castaño et al., 2015), y que nosotros también hemos aplicado con la tecnología de la realidad aumentada (Cabero y Barroso, 2018).

Por lo que se refiere a la carga cognitiva (TCC), indicar que se presentan como un marco conceptual perteneciente a las ciencias cognitivas que pretenden mejorar los ambientes tecnológicos multimedia y de formación virtual, teoría que se sitúa dentro del paradigma del procesamiento de la información. La carga cognitiva ha sido definida como "la carga que el desempeño de una tarea particular impone sobre el sistema cognitivo del aprendiz" (Paas et al., 2003, p. 64). Y como sugieren estos mismos autores, es una teoría que se ocupa del desarrollo de métodos, estrategias y medios de enseñanza que utilizan eficazmente la capacidad de procesamiento cognitivo limitado de las personas para estimular su capacidad de aplicar conocimiento y habilidades adquiridas y su transferencia (Paas et al., 2003). Dicho en otros términos la carga cognitiva se puede definir como un constructo multidimensional que representa la carga que impone una tarea particular en el sistema cognitivo del alumno. Esta teoría se basa en dos ideas comúnmente aceptadas: la primera es que hay un límite de cuánta nueva información puede el cerebro procesar a la vez; la segunda habla de que no hay límites para cuánta información "almacenada” puede ser procesada a la vez. El objetivo de la investigación en carga cognitiva es, por tanto, desarrollar técnicas de instrucción y recomendaciones que encajen con las características de la memoria de trabajo con el fin de maximizar el aprendizaje (Centre for Education Statistics and Evaluation, 2017). 
Andrade-Lotero (2012) llama la atención de que el constructo no puede ser medido directamente, y suele hacerse a través de diferentes dimensiones en concreto: la carga mental, el esfuerzo mental y el desempeño. Diferentes propuestas de instrumentos han sido realizadas por diferentes autores (Korbach et al., 2018; Naismith et al., 2015; Sweller et al., 2010; Van Gerven et al., 2002; Zumbach y Mohraz, 2008). Señalar que ya se han llevado a cabo algunos trabajos respecto a su significación en acciones de formación virtual. Incorporarla como variable independiente en nuestro estudio viene significado por que la complejidad de las tareas de aprendizaje y los contextos de aprendizaje, son variables que influyen en su concreción.

Queremos señalar que, tanto el instrumento TAM como el IMMS y el de la carga cognitiva seleccionados, serán sometidos a su fiabilización mediante la alfa de Crombach, ya que tendremos que realizar una adaptación de los mismos a los contextos tecnológicos de los MOOC.

Al mismo tiempo, a los participantes en la acción formativa se les administrará el instrumento al que anteriormente nos hemos referido para la evaluación de los contenidos por los expertos, para conocer también las percepciones y valoraciones que tienen respecto a los mismos. Su aplicación también nos servirá para conocer el grado de similitud o diferencia que se da entre los expertos y los estudiantes de cara a valorar los contenidos producidos.

Nuestra intención será también analizar las posibles relaciones que se pueden establecer entre las puntuaciones alcanzadas en el rendimiento académico y la motivación, el nivel de satisfacción mostrado en participar en la experiencia y la valoración que realizan de la experiencia.

Una vez finalizada la experiencia se realizará una entrevista en profundidad a algunos participantes con el objeto de recoger sus percepciones sobre el desarrollo de la experiencia, dificultades encontradas, dificultades que perciben que puede tener los t-MOOC para su implantación a la formación universitaria, transferencia a otra serie de contextos, etc.

También se llevarán a cabo entrevistas a aquellos participantes que hubieran abandonado la acción formativa, con el objeto de conocer los motivos de tales abandonos.

\section{CONCLUSIONES}

En este apartado se ofrecerán las principales conclusiones del estudio, en función con los datos obtenidos y la discusión realizada.

\section{REFERENCIAS}

Andrade-Lotero, L.A. (2012). Teoría de la carga cognitiva, diseño multimedia y aprendizaje: un estado del arte. Magis. Revista Internacional de Investigación en Educación, 5(10), 75-92.

Barroso, J., y Cabero, J. (2010). La investigación educativa en TIC. Visiones prácticas. Síntesis.

Cabero, J. (dir) (2018). La incorporación de las producciones polimedias a la formación universitaria. SAV de la Universidad de Sevilla.
Cabero, J. y Palacios, A. (2020). Marco europeo de competencia digital docente "DigCompEdu" y cuestionario "DigCompEdu Chek-in”. Edmetic. Revista de Educación Mediática y TIC, 9(1), 213-234.

Cabero, J., González, N., Trinidad, A.C., Ramírez, L., William, T., y Fernández, V. (2014b). Manual para el Desarrollo de la Formación Virtual - Intec Libro de Estilo. INTEC. 
Cabero, J., Llorente, MC y Vázquez, I. (2014a). Las tipologías de MOOC: su diseño e Implicaciones educativas. Profesorado. Revista de Curriculum y formación del profesorado 18(1), 13-26.

Cabero, J., y Barroso, J. (2013). La utilización del juicio de experto para la evaluación de TIC: el coeficiente de competencia experta. Bordón, 65(2), 25-38.

Cabero, J., y Barroso, J. (2018). Los escenarios tecnológicos en Realidad Aumentada (RA): posibilidades educativas en estudios universitarios. Aula Abierta, 47(3), 327-336.

Cabero-Almenara, J., Marín-Díaz, V., y Sampedro-Requena, B. (2016). Meta-analysis of research in e-learning Spanish journal published. International Journal of Educational Technology in Higher Education, 13, 25, http://dx.doi.org/10.1186/ s41239-016-0023-0.

Calvo, M.A., Rodríguez, C. y Fernández, E.M. (2016). ¿Cómo son los MOOC sobre educación? Un análisis de cursos de temática pedagógica que se ofertan en castellano. Digital Education Review, 29, 298-319. https://doi.org/10.1344/ der.2016.29.298-311

Castañeda, L., Esteve, F., y Adell, J. (2018). ¿Por qué es necesario repensar la competencia docente para el mundo digital? RED. Revista de Educación a Distancia, 56. http://dx.doi. org $/ 10.6018 / \mathrm{red} / 56 / 6$

Castaño, C., Garay, U. y Maíz, I. (2017). Factores de éxito académico en la integración de los MOOC en el aula universitaria. Revista Española de Pedagogía, 266, 65-82.

Castaño, C., Maíz, I., y Garay, U. (2014). Redes sociales y aprendizaje cooperativo en un MOOC. Revista Complutense de Educación, 26, 119-139.

Castaño, C., Maiz, I., y Garay, U. (2015). Diseño, motivación y rendimiento en un curso MOOC cooperativo. Comunicar, 44, 19-26.

Castaño, C., y Cabero, J. (2013). Enseñar y aprender en entornos m-learning. Síntesis.

Centre for Education Statistics and Evaluation (2017). Cognitive load theory: Research that teachers really need to understand. Centre for Education Statistics and Evaluation.

Chursches, A. (2008). Taxonomía de Bloom para la era digital. Eduteka. Recuperado de http://www.eduteka.org/TaxonomiaBloomDigital.php
Comisión Europea (2013). DIGCOMP: A Framework for Developing and Understanding Digital Competence in Europe. Recuperado de http://ftp.jrc.es/EURdoc/JRC83167.pdf

CRUE (2015). InformeMOOC y criterios de calidad. http:// tic.crue.org/wp-content/uploads/2016/03/InformeMOOC_ CRUETIC_ver1-0.pdf

Davies, F.D., Bagozzi, R.P., y Warsaw, P.R. (1992). Extrinsic and Intrinsic motivation to use computers in the workplace. Journal of Applied Social Psychology, 22(14), 1111-1132

Dawna, E., Stanfford, RE., Williams, KM., y Brooks, S. (2014). Evaluating the Validity and Applicability of Automated Essay Scoring in Two Massive Open Online Courses. The Internacional Review os Research in Open and Distance Learning, 15(5), 83-98.

Duart, J., (2017). La calidad pedagógica de los MOOC a partir de la revisión sistemática de las publicaciones JCR y Scopus (20132015). Revista Española de Pedagogía, 75, 29-46.

Durán, M., Gutiérrez, I., y Prendes, P. (2016a). Análisis Conceptual de Modelos de Competencia Digital del Profesorado Universitario. RELATEC. Revista Latinoamericana de Tecnología Educativa, 15(1), 172-179. Recuperado de http://relatec.unex.es/ article/view/2490

Durán, M.; Gutiérrez, I. y Prendes, M.P. (2016b). Certificación de la competencia TIC del profesorado universitario: diseño y validación de un instrumento. Revista Mexicana de Investigación Educativa, XXI, 69, 527-556 en: http://ipts.jrc.ec.europa.eu/ publications/pub.cfm?id=6359

Escudero-Nahón, A., y Núñez-Urbina, A.A. (2020). Análisis crítico al término "masivo" en los MOOC: una Cartografía Conceptual. EDMETIC, Revista de Educación Mediática y TIC, 9(1), 188212. doi: https://doi.org/10.21071/edmetic.v9i1.12252

Fernández-Díaz, E., et al. (2017). The Pedagogic Architecture of MOOC: A Research Project on Educational Courses in Spanish. International Review of Research in Open and Distributed Learning, 18(6), 18-34.

Ferrari, A. (2013). DIGCOMP: A Framework for Developing and Understanding Digital Competence in Europe. JRCQIPTS.

Fundación Telefónica (2015). Los MOOC en la educación del futuro: la digitalización de la formación. Fundación Telefónica/Ariel. 
García Barrera, A.; Gómez Hernández, P., y Monge López, C. (2017). La atención a la diversidad en los Moocs: Una propuesta metodológica. Educación XX1, 20(2), 215-233, http:// dx.doi.org/10.5944/educXX1.19038

Gisbert, M., y Lázaro, J. (2015). Professional development in teacher digital competence and improving school quality from the teachers' perspective: a case study. Journal of New Approaches in Educational Research, 4(2), 115-122.

González, A., y Carabantes, D. (2017). MOOC: medición de satisfacción, fidelización, éxito y certificación de la educación digital. RIED, 20(1), 105-123.

Guerrero, C. (2015). UMUMOOC. Una propuesta de indicadores de calidad pedagógica para la realización de cursos MOOC. Campus Virtuales, 4(2), 70-76.

Guo, Ph. et al. (2014). How MOOC Video Production Affects Student Engagement: An Empirical Study of MOOC Videos. http://pgbovine.net/publications/edX-MOOC-video-production-and-engagement_LAS-2014.pdf.

Hansch, A., Hillers, L., McConachie, K., Newman, Ch., Schildhauer T., y Schmidt, P. (2015). Video and Online Learning: Critical Reflections and Findings From the Field. HIIH Discussión Paper, 2, https://papers.ssrn.com/sol3/papers.cfm?abstract_ id $=2577882$.

Hatlevik, O. (2016). Examining the Relationship between Teachers' Self-Efficacy, their Digital Competence, Strategies to Evaluate Information, and use of ICT at School. Scandinavian Journal of Educational Research, 1-13, http://dx.doi.org/10.1 080/00313831.2016.1172501.

Hernández, R., Fernández, C., y Baptista, M. (2010). Metodología de la investigación. McGraw-Hill.

INTEF (2017). Marco común de competencia digital docente. Enero 2017. INTEF. http://blog.educalab.es/intef/2016/12/22/ marco-comun-de-competencia-digital-docente-2017-intef/.

ISTE (2008). NETS•T for Teachers: National Educational Technology Standards for Teachers, Second Edition. EUA. Recuperado de http://www.eduteka.org/pdfdir/EstandaresNETSDocentes2008.pdf.

Ju, Y., So, H., y Hee, N. (2018). Examination of relationships among students' self-determination, technology acceptan- ce, satisfaction, and continuance intention to use K-MOOCs. Computer \& Education, 122, 260-272.

Jung, Y., y Lee, J. (2018). Learning Engagement and Persistence in Massive Open Online Courses (MOOCS). Computers \& Education, 122, 9-22.

Keller, J. M. (2010). Motivational design for learning and performance. Science + Business Media, 227-286.

Korbach, A., Brünken, R. y Park, B. (2018). Differentiating Different Types of Cognitive Load: a Comparison of Different Measures. Educational Psychology Review, 30, 503-529. http:// dx.doi.org/10.1007/s10648-017-9404-8.

Lázaro, J. L., Gisbert-Cervera, M., y Silva-Quiroz, J. E. (2018). Una rúbrica para evaluar la competencia digital del profesor universitario en el contexto latinoamericano. EDUTEC, Revista Electrónica de Tecnología Educativa, 63. Recuperado de: http://dx.doi.org/10.21556/edutec.2018.63.1091.

León-Urritia, M., Cobos, R. y Dickens, K (2018). Los MOOC y su influencia en las instituciones de educación superior: perspectivas desde adentro. Journal of New Approaches in Educational Research (NAER), 7(1), 40-45. https://doi.org/10.7821/ naer.2018.1.252

López, C. (2017). ¿Cuál debería ser la longitud del vídeo educativo? Fheflippedclassroom. https://www.theflippedclassroom.es/longitud-del-video-educativo/.

López, E. (2016). En torno al concepto de competencia: un análisis de fuentes. Profesorado. Revista de currículum y formación del profesorado, 20(1), 311-322.

López-Bonilla, J. M., y López-Bonilla, L. M. (2012). Perspectiva socio-psicológica del modelo de aceptación de la tecnología. Revisión crítica del trabajo de Torres, Robles y Molina (2011). Revista Internacional de Sociología, 70(1), 205-211.

Luján, S. (2015). Barreras y estrategias de utilización de los MOOC. En P. Gómez Hernández, A. García Barrera, C., y Monge López (Edit), La cultura de los MOOC (pp. 141.160). Síntesis.

Marauri, P. M. (2014). La figura de los facilitadores en los Cursos Online Masivos y Abiertos (COMA/MOOC): nuevo rol profesional para los entornos educativos en abierto. RIED. Revista Iberoamericana de Educación a Distancia, 17(1), 35- 67. 
Margaryan, A., Blanco, M., y Littlejohn, A. (2015). Instructional quality of Massive Open Online Courses (MOOCs). Computers \& Education, $80,77-83$.

Martí, J. (2012). Tipos de MOOCs. Xarxatic. Disponible en: http:// www.xarxatic.com/tipos-de-moocs/.

Mengual, A., y Roig, R. (2015). Validación del Cuestionario de evaluación de la calidad de cursos virtuales adaptado a MOOC. RIED, 18(2), 145-169.

Meseguer, A., Ros-Gálvez, A. y Rosa-García, A. (2017). Satisfaction with online teaching videos: A quantitative approach. Innovations in Education and Teaching International, 54(1), 62-67, https://doi.org/10.1080/14703297.2016.1143859.

Naismith, L., Cheung, J., Ringsted, Ch. y Cavalcanti, R. (2015) Limitations of subjective cognitive load measures in simulation-based procedural training. Medical Education, 49 $805-814$

Osuna-Acedo, S. Marta-Lázaro, C., y Faru-Meigs, D. (2018). De sMOOC a tMOOC, el aprendizaje hacia la transferencia profesional: El proyecto europeo ECO. Comunicar, 55, 105-114.

Paas, F., Tuovinen, J., Tabbers, H., y Van Gerven, P. (2003). Cognitive Load Measurement as a Means to Advance Cognitive Load Theory. Educational Psychologist, 38(1), 63-71, https:// doi.org/10.1207/S15326985EP3801_8.

Quiliano-Terreros, R., Ramirez-Hernández, D., y Barnoil, P. (2019). Systematic mapping study 2012-2017: quality and effectiveness measurement in MOOC. Turkish Online Journal of Distance Education-TOJDE, 20(1), 223-247.

Rangel, A. (2015). Competencias docentes digitales: propuesta de un perfil. Pixel-Bit. Revista de Medios y Educación, 46, 235-248. Ruiz-Palmero, J., López-Álvarez, D., Sánchez-Rivas, E. y SánchezRodríguez, J. (2019). An Analysis of the Profiles and the Opinion of Students Enrolled on xMOOCs at the University of Málaga. Sustainability, 11, 6910; https://doi.org/10.3390/ su11246910.

Sánchez, M. M. y Prendes, M. P. (2015). Más allá de las pruebas objetivas y la evaluación por pares: Alternativas de evaluación en los MOOC. RUSC. Revista de Universidad y Sociedad del Conocimiento, 12(1), 1-14. Recuperado de http://rusc.uoc. edu/rusc/es/index.php/rusc/article/view/v12n1-sanchezprendes-espinosa/2468.html.
Sánchez, M. M., González, V., y Prendes, M. P. (2017). Los MOOC y la evaluación del alumnado: revisión sistemática (20122016). @TIC Revista de Innovación Educativa, (18), 65-74. https://doi.org/10.7203/attic.18.10013.

Sánchez, Ma , González-Calatayud, V., y Prendes-Espinosa, M. P. (2017). Los MOOC y la evaluación del alumnado: revisión sistemática (2012-2016). @tic. revista d'innovació educativa, 18, $65-73$.

Scopeo (2013). SCOPEO INFORME N². MOOC: Estado de la situación actual, posibilidades, retos y futuro. Universidad de Salamanca-Centro Internacional de Tecnologías Avanzadas.

Sosa, A., Salinas, J., y De Benito, B. (2018). Las tecnologías emergentes en las actividades de aprendizaje al implementar un modelo de incorporación de tecnología en el aula. European Journal of Education Studies, 4(1), 155-173. https://doi. org/10.5281/zenodo.1158667

Sosa, M. J. y Fernández, M. R. (2015). Análisis de las oportunidades y problemas que ofrecen los MOOC, en Gómez, P., et al. (eds). La cultura de los MOOC (pp. 161-197). Síntesis.

Sweller, J., Ayres, P., y Kalyuga, S. (2011). Cognitive Load Theory Springer Science+Business Media.

The Scottish Government (2016). Enhancing learning and teaching through the use of digital technology. The Scottish Government.

Tourón, J., Martín, D., Navarro, E., Pradas, S., e Îñigo, V. (2018). Validación de constructo de un instrumento para medir la competencia digital de los profesores (CDD). Revista Española de Pedagogía, 269, 25-54.

Unesco (2008). Estándares de competencia en TIC para docentes. Recuperado de http://www.eduteka.org/EstandaresDocentesUnesco.php.

Unesco (2016). Tecnologías digitales al servicio de la calidad educativa. Unesco: Santiago de Chile.

Van Gerven, P., Paas, F., Van Merriënboer, Schmidt, H. (2002). Cognitive load theory and aging: effects of worked examples on training efficiency. Learning and Instruction, 12, 87-105.

Vázquez, E., et al (2013). La expansión del conocimiento abierto: los MOOC. Octaedro. 
Watted, A., y Barak, M. (2018). Motivating factors of MOOC completers: Comparing between universityaffiliated students and general participants. The Internet and Higher Education, 37, 11-20.

Wojciechowski, R., y Cellary, W. (2013). Evaluation of learners' attitude toward learning in ARIES augmented reality environments. Computers \& Education, 68, 570-585.

Zhou, M. (2016). Chinese university students' acceptance of MOOCs: A selfdetermination perspective. Computers \& Education, 92-93, 194-203.

Zumbach, J. y Mohraz, M. (2008). Cognitive load in hypermedia reading comprehension: Influence of text type and linearity. Computers in Human Behavior, 24, 875-887.

PROYECTO I+D+i Retos Investigación-2018

DISEÑO, PRODUCCION Y EVALUACION DE T-MOOC PARA LA ADQUISICION POR LOS DOCENTES DE COMPETENCIAS DIGITALES DOCENTES - DIPROMOOC- Ref.-RTI2018-097214-B-C31. Ministerio de Ciencia, Innovación y Universidades 